\title{
Subgrain dynamics during recovery of partly recrystallized aluminium
}

\author{
Ahl, S. R.; Simons, H.; Detlefs, C.; Juul Jensen, D.; Poulsen, H. F.
}

\section{Published in:}

Acta Materialia

Link to article, DOI:

10.1016/j.actamat.2019.10.042

Publication date:

2020

Document Version

Peer reviewed version

Link back to DTU Orbit

Citation (APA):

Ahl, S. R., Simons, H., Detlefs, C., Juul Jensen, D., \& Poulsen, H. F. (2020). Subgrain dynamics during recovery of partly recrystallized aluminium. Acta Materialia, 185, 142-148. https://doi.org/10.1016/j.actamat.2019.10.042

\section{General rights}

Copyright and moral rights for the publications made accessible in the public portal are retained by the authors and/or other copyright owners and it is a condition of accessing publications that users recognise and abide by the legal requirements associated with these rights.

- Users may download and print one copy of any publication from the public portal for the purpose of private study or research.

- You may not further distribute the material or use it for any profit-making activity or commercial gain

- You may freely distribute the URL identifying the publication in the public portal 


\section{Journal Pre-proof}

Subgrain dynamics during recovery of partly recrystallized aluminium
S.R. Ahl , H. Simons,
C. Detlefs,
D. Juul Jensen, H.F. Poulsen

PII:

DOI:

S1359-6454(19)30704-9

Reference:

To appear in:

Received date:

Revised date:

Accepted date: https://doi.org/10.1016/j.actamat.2019.10.042

AM 15610

\section{Acta Materialia}

18 April 2019

20 October 2019

24 October 2019

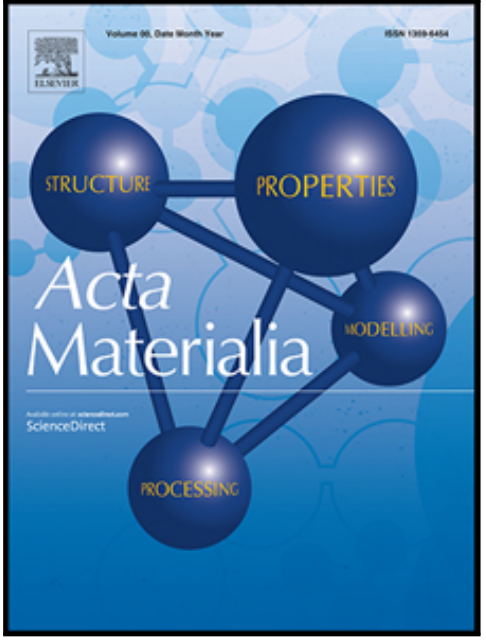

Please cite this article as: S.R. Ahl, H. Simons, C. Detlefs, D. Juul Jensen, H.F. Poulsen, Subgrain dynamics during recovery of partly recrystallized aluminium, Acta Materialia (2019), doi: https://doi.org/10.1016/j.actamat.2019.10.042

This is a PDF file of an article that has undergone enhancements after acceptance, such as the addition of a cover page and metadata, and formatting for readability, but it is not yet the definitive version of record. This version will undergo additional copyediting, typesetting and review before it is published in its final form, but we are providing this version to give early visibility of the article. Please note that, during the production process, errors may be discovered which could affect the content, and all legal disclaimers that apply to the journal pertain.

(C) 2019 Published by Elsevier Ltd on behalf of Acta Materialia Inc. 


\title{
Subgrain dynamics during recovery of partly recrystallized aluminium
}

\author{
S.R. Ahl' ${ }^{1}$, H. Simons ${ }^{1}$, C. Detlefs ${ }^{2}$, D. Juul Jensen ${ }^{3}$ and H. F. Poulsen ${ }^{1}$ \\ 'Department of Physics, Technical University of Denmark, 2800 Kgs Lyngby, Denmark \\ ${ }^{2}$ European Synchrotron Radiation Facility, 71 Avenue des Martyrs, 38000 Grenoble, France \\ ${ }^{3}$ Department of Mechanical Engineering, Technical University of Denmark, 2800 Kgs Lyngby, Denmark
}

Keywords: Recovery, synchrotron, high-energy X-ray diffraction, aluminium, subgrain growth

\begin{abstract}
The recovery dynamics of 498 individual subgrains within the non-recrystallized matrix of a $50 \%$ recrystallized aluminium sample is studied using high resolution X-ray diffraction at a synchrotron source. The subgrains' change in size, orientation, axial strain and internal disorder are monitored as functions of temperature. The subgrains are surprisingly active, and the individual subgrains exhibit markedly different growth kinetics. Contradictory to common knowledge we find that the individual subgrains' sizes change significantly even though the average size remain constant. Furthermore we observe no correlation between subgrain size and growth rate. An analysis of the volume changes of the individual subgrains indicates a broad spectrum of critical temperatures above which the subgrains grow or shrink. Possible mechanisms are discussed and related to relaxation of local stresses.
\end{abstract}

\section{Introduction}

When a deformed metal is annealed, the microstructure changes by processes generally referred to as recovery and recrystallization. During recrystallisation the microstructure is changed by the formation and growth of nearly defect free nuclei, leading to a significant softening of the material. Recovery is typically associated with much more subtle changes in microstructure and far less softening [e.g. 1, 2]. This may explain why recovery has attracted far less attention than recrystallization. There are, however, cases when recovery leads to softening of almost the same magnitude as recrystallization [e.g. 3, 4]. Altogether, recovery is of utmost importance for the mechanical properties and for the subsequent recrystallization which is driven by a stored energy, which is lower than that being present in the material in the deformed state.

Recovery is generally defined as all annealing processes occurring in deformed materials which do not involve long range migration of high angle boundaries (with misorientations $>15^{\circ}$ ) [5]. This is occurring by a range of processes including dislocation annihilation by glide, climb and cross slip as well as absorption of dislocations at grain boundaries. The relative importance of these processes depends on the material, strain, strain rate and temperature [e.g. 6, 7]. In some publications also subgrain growth by boundary migration [2] and triple junction motion [8, 9] are demonstrated as recovery processes. Consequently, recovery is associated by many activation energies, and already in 1948, Kuhlman suggested that the apparent activation energy increases as recovery proceeds [10]. This is discussed in detail in [2] and experimentally documented by [11]. The increase in activation energy is related to the decrease in stored energy in the recovering matrix and values up to the activation energy for recrystallization are observed [12]. At that stage recrystallization "takes over" and the recovered microstructure is obliterated by recrystallization.

Very few studies have focused on the possibility of recovery occurring in the non-recrystallized matrix concurrent with recrystallization and the results are non-conclusive. For example in Fe, Vandermeer and Rath found that the stored energy in the non-recrystallized matrix decreases [13], while others found that in Cu the matrix is stable [14] and in Al the misorientation between recrystallizing grains and the matrix is observed to be constant during recrystallization [15].

The aim of the present work is therefore to study if recovery continues in a sample that is already partly recrystallized. We use a newly developed X-ray diffraction method, which provides unique information on the dynamics of the individual subgrains in the non-recrystallized matrix.

Classical $\mathrm{x}$-ray powder diffraction gives rise to continuous rings on a 2D detector and can provide accurate information on size and strain properties but only as averages over the microstructure. By contrast, 3 Dimensional X-ray diffraction (3DXRD) monitors the diffraction spots from individual grains. 3DXRD can be performed in modes that provide 3D volumetric mapping of the grains or alternatively in fast data acquisition modes for providing statistics on the properties of individual grains during processing [16]. Lauridsen et al. used the latter mode to identify and track spots from several hundred bulk recrystallizing grains over time during annealing $[17,18]$. From such measurements Poulsen et al. determined individual growth rates and activation energies [19]. Being near perfect crystals, the subgrains in a well recovered matrix also give rise to diffraction spots although of lower intensity than the recrystallizing grains. However, carrying out the above type of 3DXRDmeasurements on the subgrain structures is difficult due to the vast number of illuminated subgrains and corresponding vast number of overlapping diffraction spots. At present, the maximum number of identifiable diffracting units in a 3DXRD experiment is a few thousand [20], which limits the sample size for a recovery experiment to a thin plate less than $100 \mu \mathrm{m}$ thick even for a micron-sized point-beam. By using a thin film sample, the feasibility of tracking individual subgrains during annealing was demonstrated by Gundlach et al. [21].

In the present work, the 3DXRD fast acquisition approach was applied and the setup was altered to allow tracking of subgrains in mm thick specimens at the expense of only characterizing a section of orientation space. In this work 2000 individual diffraction spots were identified and their characteristics interpreted in terms of subgrain size and internal disorder. Furthermore, each subgrain was tracked during annealing at a constant heating rate and the dynamics was quantified in terms of growth rate, as well as changes in strain and orientation, leading to a statistical investigation of the recovery happening at annealing stages when also recrystallization is on-going elsewhere in the sample.

Cold rolled aluminium of commercial purity (AA1050) was chosen for the experiment. For this material it is well known that even the as-rolled microstructure is dynamically recovered and significant softening occurs by recovery prior to recrystallization [4]. Our hypothesis was thus that in a $50 \%$ recrystallized sample, the subgrain structure in the non-recrystallized matrix would be rather stable. As shall be shown, our hypothesis was however, found to be false. The new measurements show that even at annealing temperatures below the recrystallization temperature lots of activities are found in the not yet recrystallized matrix in the $50 \%$ recrystallized sample.

The experimental data point to the need for new recovery analysis and are essential for interpretation of the recrystallization processes and kinetics. 


\section{Material and experimental procedure}

The material used for the present work was commercially $99.5 \%$ pure aluminium (AA1050) with an initial grain size of $70 \mu \mathrm{m}$. This material was cold rolled to $50 \%$ reduction in thickness and annealed for 50 minutes at $325^{\circ} \mathrm{C}$ in an air furnace. The furnace was at temperature when the sample was put in, and after annealing, the sample was air cooled. Thereby the sample was partly recrystallized and the volume fraction recrystallized was found to be $50 \%$ by Electron Back Scattering Diffraction (EBSD). Using electrical discharge machining, a rod-shaped sample was cut out, with a cross-sectional area of $300 \times 300 \mu \mathrm{m}^{2}$ perpendicular to the rolling direction (RD) and a length of $1 \mathrm{~mm}$.

The novel experimental approach used is a high spatial resolution version of 3DXRD and is somewhat similar to that presented in [17, 18]. The main difference to classical 3DXRD is that the far field 2D detector, with typical pixel sizes of 50-100 $\mu \mathrm{m}$, is replaced by a near field $2 \mathrm{D}$ detector with an effective pixel size below $1 \mu \mathrm{m}$. As shown in the sketch of the experimental geometry in Figure 1a, this detector is placed off the optical axis. Due to the limited field of view of the detector, only a section of a single Debye-Scherrer ring is acquired. Moreover, the sample is only rotated through a few degrees instead of a full $180^{\circ}$ or $360^{\circ}$ rotation. As a result only a sub-set of subgrains are monitored, corresponding to a small region within a pole figure. These modifications allow recording of thousands of subgrain in thick bulk samples.

The experiment was performed at the Dark Field X-ray Microscopy (DFXM) beamline ID06 at the European Synchrotron Radiation Facility (ESRF). Using a Si-111 Bragg-Bragg monochromator, a $16 \mathrm{keV}$ monochromatic X-ray beam was generated. This was focused and defined by slits to provide a uniform box beam on the sample of size $100 \times 100 \mu \mathrm{m}^{2}$.

The sample was placed immediately after the slits at a distance of $55 \mathrm{~m}$ from the source on a goniometer that allowed high precision control over tilts and translations of the sample. For the present experiment we used only the 'rocking angle', i.e. the rotation $u$ around the horizontal axis perpendicular to the beam direction, see Figure 1a.

The near field detector is an indirect 2D detector composed of a scintillator screen, an optical microscope and a FreLon CCD camera [22]. The detector has an effective pixel size of $0.622 \mu \mathrm{m}$ corresponding in the vertical direction to a resolution of $\Delta 2 \theta=0.0014^{\circ}$ and in the horizontal direction to $\Delta \eta=0.0047^{\circ}$ (for definition of angles see Figure 1a). The spatial resolution is matched to the effective pixel-size. This detector was placed at a distance of $22 \mathrm{~mm}$ downstream of the sample position and elevated with respect to the direct beam to collect (111)-reflections at $2 \theta$ $=19.1^{\circ}$. At this position the detector covered a section of the Debye-Scherrer ring equivalent to $\Delta \eta=9.5^{\circ}$ with a vertical width related to the thickness of the sample, see Figure 1a. To avoid surface effects, a single deformed grain in the middle of the sample was identified and used.

The sample was heated continuously with a hot air blower from $25^{\circ}$ to $75^{\circ}$ at a constant heating rate of initially $1.2^{\circ} \mathrm{C} / \mathrm{min}$, and then of $0.6^{\circ} \mathrm{C} / \mathrm{min}$ up to $225^{\circ}$. It has to be noted that even the maximum temperature in this experiment is thus $100^{\circ}$ lower than that of the previous annealing. The reasons for choosing this temperature range for the present investigations was the significant and unexpected activity in subgrain structure observed while heating the sample. The duration of the fulli experiment was 5 hours. The actual sample temperature was determined with an accuracy better than $10^{\circ} \mathrm{C}$ from the aluminium lattice expansion, i.e. from the vertical shift of the total $2 \theta$-ring. Movement and rotation of the sample during the annealing due e.g. to expansion of the sample holder was accounted for in the data analysis software. However, the actual shift in the gauge volume implied that the number of subgrains traceable during the entire annealing was significantly reduced from a few thousand to about five hundred.

While heating, data series were collected in situ for a total of 117 temperatures in the interval, each consisting of 40 consecutive images covering a tilt range of $\pm 1^{\circ}$ in $u$ in steps of $0.05^{\circ}$. Each image was acquired while the sample was rotated continuously through the $0.05^{\circ}$ step. Combining all 40 images in a data series reveals several thousand diffraction spots. In Figure $1 \mathrm{~b}$ an example of such a combined data set is shown with colors indicating tilt in $u$ and brightness indicating the diffracted intensity at that point. The internal mosaic spread of the subgrains was less than the step size of $0.05^{\circ}$.

While individual diffraction spots from several thousand subgrains were identified at each temperature, the movement and rotation of the sample reduced this number to 498 individual spots which could be traced through the series from $80^{\circ} \mathrm{C}$ to $225^{\circ} \mathrm{C}$. For each of these the center position in rocking angle $u$, the central position on the detector $\left(x_{d}, z_{d}\right)$, the integrated intensity, $I_{s}$, and the area of the peak, $A$, were determined after a temperature-dependent background and collective shifts had been corrected for.

The integrated intensity of a diffraction spot can be related to subgrain volume, $V_{s}=\frac{4 \pi}{3} R_{e q}^{3}$, with $R_{e q} \propto \sqrt[3]{I_{s}}$ being the equivalent sphere radius [18]. The ratio $W=\frac{A}{\pi R_{e q}^{2}}$ is taken as a crude measure of the disorder within a given subgrain since the peak area is influenced by both the size and the internal mosaicity of the corresponding subgrain. $u$ is a direct measure of the tilt of the (111)-lattice plane normal, see Figure 1a. The position on the detector, $\left(x_{d}, z_{d}\right)$, is determined by a combination of the position of the subgrain within the sample, its orientation and its average strain. We cannot from a single image de-convolve this information. However, the changes in subgrain radii were in general only small, corresponding to less than a pixel, see below. Thus the observed shifts in position on the detector could not be accounted for by spatial translation of the subgrain center-of-mass. Therefore, the observed horizontal shifts in spot position are ascribed to lattice rotation and vertical shifts to changes in lattice spacing.

\section{Results}

The statistical analysis of the dynamics of the 498 individual subgrains is summarized in Figure 2. The first column shows the evolution with temperature of the size, disorder, subgrain rotation and axial strain for 10 randomly selected subgrains. The statistic for all the 498 subgrains are shown in the middle column, with the central line being the average and the surrounding lines being one and two standard deviations, respectively. The right column shows the distributions at selected temperatures.

The evolution in size of individual subgrains was tracked during heating (Figure 2a). None of these growth curves are identical and individual subgrains may grow in one temperature interval and shrink in another. Some changes happened smoothly and continuously over larger temperature intervals, other changes were more abrupt.

As can be seen in Figure $2 \mathrm{~b}$ the average subgrain size of $R_{e q} \sim 1 \mu \mathrm{m}$ does not change significantly during the annealing. The lower threshold of $\sim 200 \mathrm{~nm}$ indicates that all illuminated subgrains in the monitored section of orientation space were detected. Also, the shapes of the 
distributions (Figure 2c) are found to be largely similar for all temperatures. Thus in general, the average behavior and the size distributions do not reflect the diversity and amplitude in the growth behavior of the individual subgrains.

The internal disorder, as quantified by the disorder parameter $W$, also varies as a function of temperature for the individual subgrains (Figure 2d), while the average remains largely constant (Figure 2e). This means that on average the mosaicity within the subgrains is not changing, but as a given subgrain is growing or shrinking its internal mosaicity may change significantly. It either decreases or increases depending on the local rearrangements of dislocations within that subgrain as it grows or shrinks. For example, the disorder parameter of a shrinking subgrain will increase if the number of dislocations is preserved so that they are crammed in the subgrain interior to increase the local defect density. Likewise, $W$ decreases if dislocations annihilate or move to the subgrain boundaries to reduce the defect density within the subgrain. Such a behavior could explain the weak peak seen in Figure $2 e$ around $180^{\circ} \mathrm{C}$, see below, although the observed effect is too small to be conclusive.

On average the shift in horizontal detector position with respect to position at $80^{\circ} \mathrm{C}$ was zero (Figure $2 \mathrm{~h}$ ) while the distribution of shifts (Figure 2i) broadened with increasing temperature to become a symmetric double peak at $222^{\circ} \mathrm{C}$. The double peak corresponds to a lattice rotation of $\sim 0.014^{\circ}$ which could relate to either a pure lattice rotation or a shear strain of $2.4 \cdot 10^{-4}$ (or a combination of the two). For a spherical subgrain of radius $1 \mu \mathrm{m}$ the observed rotation corresponds to atoms near the boundary moving $2.5 \AA$ along the boundary, similar to the interatomic distance in the structure. For comparison, a single edge dislocation (Burgers vector $b=2.335 \AA$ ) through the center of a subgrain of $1 \mu \mathrm{m}$ radius causes a difference in lattice orientation of $0.013^{\circ}$ from side to side. Thus rotations of a similar magnitude are expected if a dislocation enters or leaves subgrains of the observed average size.

Also the vertical shift distribution (Figure 2l) broadens with temperature and exhibits a shoulder at $\sim 3$ pixels above $160^{\circ} \mathrm{C}$ and symmetric side peaks at shifts of 7 pixels for the highest temperatures. The positive mean shift in vertical detector position indicates that the individual trackable subgrains on average expanded less with increasing temperature than the original grain as a whole. It is not possible from these measurements to determine whether negative axial strain is building up due to external pressure on these subgrains or whether initially present positive strain is released.

The side peaks in the vertical shift distribution corresponds to compressive/tensile changes in axial strain of $\varepsilon \sim 5.3 \cdot 10^{-4}$. For a spherical subgrain of $1 \mu \mathrm{m}$ radius such a change in axial strain means a lattice expansion or contraction that corresponds to atoms near the subgrain boundary moving $5.3 \AA$ along the radial direction, again similar to the interatomic distances in the sample. Notably, only strain changes are observed in the experiment, so both of these side peaks may represent strain relaxation.

As the observed rotations were smaller than the step size in $u$, similar lattice rotations in $u$ could not be observed in the present experiment, although the position in $u$ was a direct measure of lattice orientation. As discussed above all shifts in detector position were interpreted in terms of lattice rotations and changes to internal strain since the observed changes in subgrain sizes were too small to generate center-of-mass translations large enough to explain the observed shifts. Thus based on this interpretation the data indicate that subgrain coalescence is not a dominating mechanism in the present experiment.

\subsection{Growth rates}

The volumetric growth rate for each subgrain was calculated as the derivative of the subgrain volume $V_{S}$ versus temperature: $v_{T}=\frac{d V_{s}}{d T}$. For each temperature the mean growth rate and the associated standard deviation for growing and shrinking subgrains are shown in Figure $3 a$. The mean growth rate exhibits a peak at $160^{\circ} \mathrm{C}$. This is consistent with the distribution of growth rates (Figure $3 \mathrm{~b}$ ) being slightly broader at $157^{\circ} \mathrm{C}$, indicating faster dynamics at this temperature. This suggests that a thermally activated process became dominant around this temperature. However, since the average size does not change markedly, this process merely caused a rearrangement of boundaries, not an actual overall coarsening of the structure.

A scatter plot of growth rate versus subgrain volume is shown in Figure 3c. No clear correlation between subgrain size and growth rate is evident. This is confirmed by a statistical test based on the ensemble of all subgrains: the Kendall $T$-coefficient is approximately zero, see Figure 3d. A scatter plot of growth rate versus disorder is shown in Figure $3 \mathrm{e}$ and the corresponding statistical correlation in Figure $3 \mathrm{f}$ which reveals no significant correlation. Thus within the statistical uncertainty of these tests the subgrain size and disorder does not determine whether a subgrain is growing or shrinking. However, Figs $3 \mathrm{~d}$ and $3 \mathrm{f}$ may indicate that larger, more uniform subgrains may be more likely to exhibit faster changes in size, which calls for further investigation.

\section{Discussion}

\subsection{Experimental outlook}

Characterizing the evolution of the microstructure of deformed metals non-destructively in 3D is a challenge. Existing non-destructive X-ray diffraction imaging methods such as 3DXRD and diffraction contrast tomography (DCT) are limited to deformations below $10 \%-20 \%$, due to the broadening of diffraction spots from deformed grains. Furthermore the spatial resolution is too poor to map the subgrains. Other methods such as Dark Field X-ray Microscopy [23-25] and Differential Aperture X-ray Microscopy [26-28] have a spatial resolution that allows mapping of subgrains, but these methods are limited in terms of the number of subgrains characterized. As we have shown here, it is important to sample a sizable population of subgrains for the present type of investigation.

In the approach presented here, the spot overlap issue of 3DXRD and DCT is overcome by resolving the subgrains. The subgrain diffraction spots exhibit almost no angular spread (in this case less than $0.05^{\circ}$ ), and hence can be separated by placing a high resolution detector at a sufficiently large distance to the sample. With a comprehensive characterization of thousands of subgrains within 2 minutes, statistics on very large ensembles of subgrains is possible by translating or rotating the sample. Moreover the cutoff in terms of subgrain size - which is inherent to any method based on segmentation - is a factor of 5 below the typical average subgrain size of $1 \mu \mathrm{m}$ [6], implying that the sampling is essentially unbiased. By increasing the coverage on the $(u, \eta)$ diffraction sphere, the ensemble could therefore be extended to all subgrains belonging to a given deformed grain. 
recrystallized grains and DFXM for mapping the shape, mosaicity and strain inside individual subgrains or recrystallizing grains with high resolution.

\subsection{Analysis of the recovery}

During the experiment no significant microstructural coarsening took place, since the size distribution and mean equivalent radius of the subgrains remained largely unchanged, see Figure 2. Nevertheless, the size and disorder parameter for individual subgrains evolved with temperature, as did the shear and axial strain. Previous studies have shown that individual subgrains evolve differently related to local stored energy differences [29, 30]. However, these previous studies were focusing on recovery before recrystallization. The present result is therefore unique as it documents significant local activation of recovery processes even at late recrystallization stages. The evolution is, in the present experiment, observed not to be smooth, but peaks at certain temperatures. Significant variations in the disorder parameter for the individual subgrains were observed in the range $140-180^{\circ} \mathrm{C}$. Furthermore, at $160^{\circ} \mathrm{C}$ the growth rates were largest. This reflects the local behavior of a given ensemble of subgrains originating from the same deformed grain. Such "transition" temperatures may be interpreted as local thermal activation of a certain dislocation annihilation mechanism at that temperature.

It is customary to quantify recovery in terms of a bulk property such as hardness, and average subgrain size [2] and investigate the evolution of this property with temperature or annealing time. However, the present study reveals that the average subgrain size was almost constant although the sizes of individual subgrains change markedly. Notably within the temperature range explored, no correlation between subgrain size and growth rate was observed. This is contradicting classical recovery models, which generally assume that large subgrains grow while the smaller ones shrink [7]. Also, abnormal growth of some subgrains has been observed by electron microscopy [31] which may lead to nucleation of recrystallization. Our observations imply that at the investigated late state of recovery the microstructural rearrangements are more minor in scale and the driving force is of a more local nature, presumably related to the local dislocation density and morphology or the subgrain boundary misorientation (which were not observed in this experiment). It should furthermore be noted that all the characterized subgrains have more or less the same crystallographic orientation and are from a single original grain, which means that they originate from more or less the same set of slip systems operating during the deformation. The observed dynamics thus appear to be determined by the internal strain (equivalent to residual stress) and remaining dislocation density causing disorder in individual subgrains. Even though only relatively few dislocations remain inside the subgrains and/or near the subgrain boundaries at this late annealing stage $(50 \%$ recrystallized aluminium) the dynamics of these seem to have huge influence on the evolution of the hosting subgrain making it grow or shrink. This interpretation partly matches that of Hasegawa et al. [32] who in an investigation of pure aluminium single crystals, suggest Type II (or 'true') recovery at later stages of recovery leading to tightening of the cell walls into subgrain boundaries and to subgrain coarsening. Our results reveal dislocation activities within the subgrains and/or near subgrain boundaries leading to changes in the individual subgrain size (a subgrain may grow or shrink) driven by the remaining residual stress/strain, whereas we do not observe an overall subgrain coarsening in the present experiment. It is furthermore likely that where one or a few dislocations are annihilated in a given subgrain or in the subgrain boundary, it may release its 'pinning effect' also in the neighbor subgrain and thus cause a short range relaxation reaction. Altogether this contributes to lowering the energy stored in the yet non-recrystallized matrix.

It is intriguing that subgrain recovery occur to the observed extent in a 50\% recrystallized sample at temperatures much lower than the recrystallization temperature, and it may be questioned if there is a sufficient number of dislocations still present in the non-recrystallized parts of the structure (in the subgrain interior as well as in the subgrain boundaries) which together with remaining local residual stress can drive further recovery. The present experimental observations show that this is the case, and our result is substantiated by previous synchrotron experiments showing that even in the interior of the recrystallized grains, there is a low density of dislocations organized mostly in very low angle boundaries, giving rise to measurable mosaicity [33] as well as to the presence of residual strain [34]. Also, it may be questioned if the small sample size and thus near-surface effects such as scratches and additional strains related to the sample preparation may have affected the results. As the present measurements are from a gauge volume near the middle of the sample, see Figure 1a, and no signs of recrystallization are observed, e.g. by growth of surface stimulated nuclei, we suggest that the present results are typical for bulk subgrains of the present conditions also in much larger samples.

The present findings prove that the evolution of the average subgrain size is not an adequate measure of the local recovery reactions. Instead, the dynamics of individual subgrains and the evolution of their internal strain and dislocation density should be monitored to quantify the overall processes via statistics.

It has to be underlined that the present measurements are monitoring subgrains in the bulk of the sample. The small strains, which are observed to remain within the subgrains, are likely to be relieved at free sample surfaces. It is therefore crucial to study the bulk kinetics to allow for the importance of the remaining residual strains.

\section{Conclusions}

A high spatial resolution variant of 3DXRD has been developed and used to quantify the dynamics of individual subgrains during recovery in the non-recrystallized matrix of an aluminium (AA1050) which prior to the experiment was $50 \%$ recrystallized. At this annealing stage the subgrains are nearly perfect, containing only a few interior dislocations each.

Several thousand such subgrains have been identified from a series of high angular resolution X-ray diffraction images and characterized in terms of size and internal disorder, subgrain rotation and strain while heating at a constant rate to $225^{\circ} \mathrm{C}$. Furthermore, the kinetics of 498 individual subgrains has been tracked during the entire heating interval. It is found that:

- The average subgrain size and the size distribution remain constant during the annealing expressing that on average the not yet recrystallized microstructure is stable.

However, the individual subgrains grow and shrink significantly. Each individual subgrain has its own growth kinetics, which is markedly different for different subgrains and vary with temperature.

- No statistically significant correlation is observed between subgrain size and growth rate. This observation is in contradiction with classical models for recovery kinetics, which assume a direct correlation between size and growth rate.

No subgrain lattice rotations larger that the experimental step size of $0.05^{\circ}$ was observed, documenting that subgrain coalescence is not an important recovery mechanism in the present experiment. 
- The average internal disorder parameter remains largely constant while that of the individual subgrains evolve during the annealing. This suggests perfection of the dislocation and defect structure within individual subgrains and at the boundaries, affecting also the neighboring subgrains.

- Transitions in the internal disorder parameter and in the growth rate evolution suggest activation of different dislocations annihilation mechanisms at different temperatures.

- On average the axial strain is largely constant but within the distribution, individual subgrains exhibited a strain change by up to $5 \cdot 10^{-4}$. Likewise, the shear strain evolved with evidence for a preferred accumulated strain change of $2 \cdot 10^{-4}$. We interpret these strain changes as relaxations reducing medium / long range stresses and suggest these to be the main driving force for the observed structural dynamics.

\section{Acknowledgements}

SRA, HS and HFP acknowledge an ERC Advanced Grant no 291321 - d-TXM. DJJ acknowledge funding from the European Research Council (ERC) under the European Union's Horizon 2020 research and innovation programme (M4D - grant agreement No 788567). We thank ESRF for provision of beamtime and the Danish instrument center Danscatt for a travel grant. CD acknowledges financial support from the Otto Mønsteds fond. We are grateful to the ID06 team for technical advice and experimental help.

Declaration of Competing Interest

The authors declare that they have no known competing financial interests or personal relationships that could have appeared to influence the work reported in this paper.
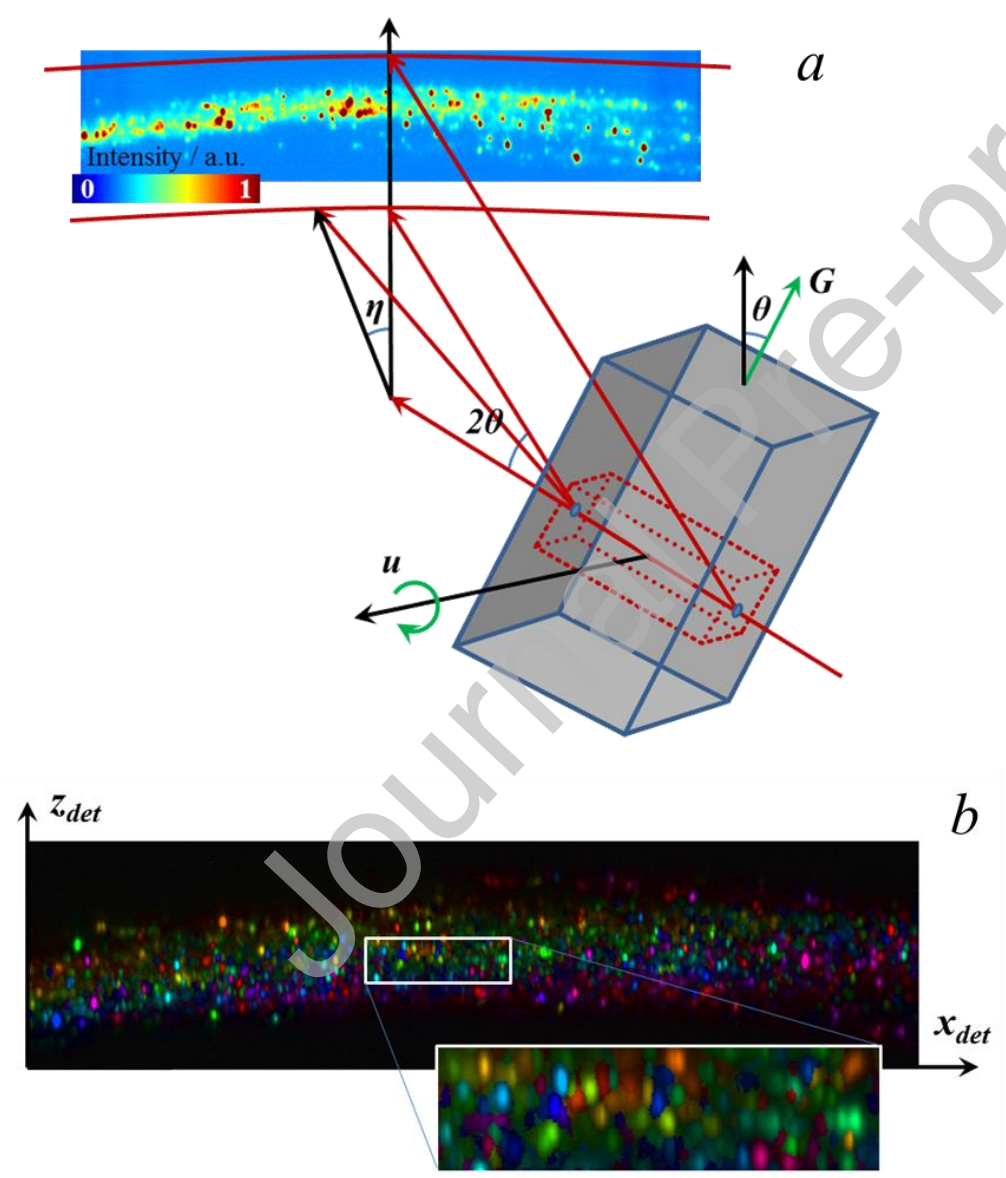

Figure 1: a) Experimental setup. Diffraction from subgrains within the gauge volume (marked as a box with dashed lines) gives rise to spots on a high resolution detector within a band in scattering angle $2 \theta$ and a section $\Delta \eta$ of the (111) Debye-Scherrer ring. Exposures were made as function of tilt angle $u$. An example of a raw data image is shown with intensity in arbitrary units. The vertical position on the detector is primarily determined by the position within the sample in the direction of the thickness. b) A combined image representing information from the entire $u$ range. The brightness of each pixel represents the maximum intensity for that pixel and the color represents the orientation in $u$ for which the intensity was maximum. The insert shows a zoom on the region marked by the white box.

[ Figure 1 has been updated] 

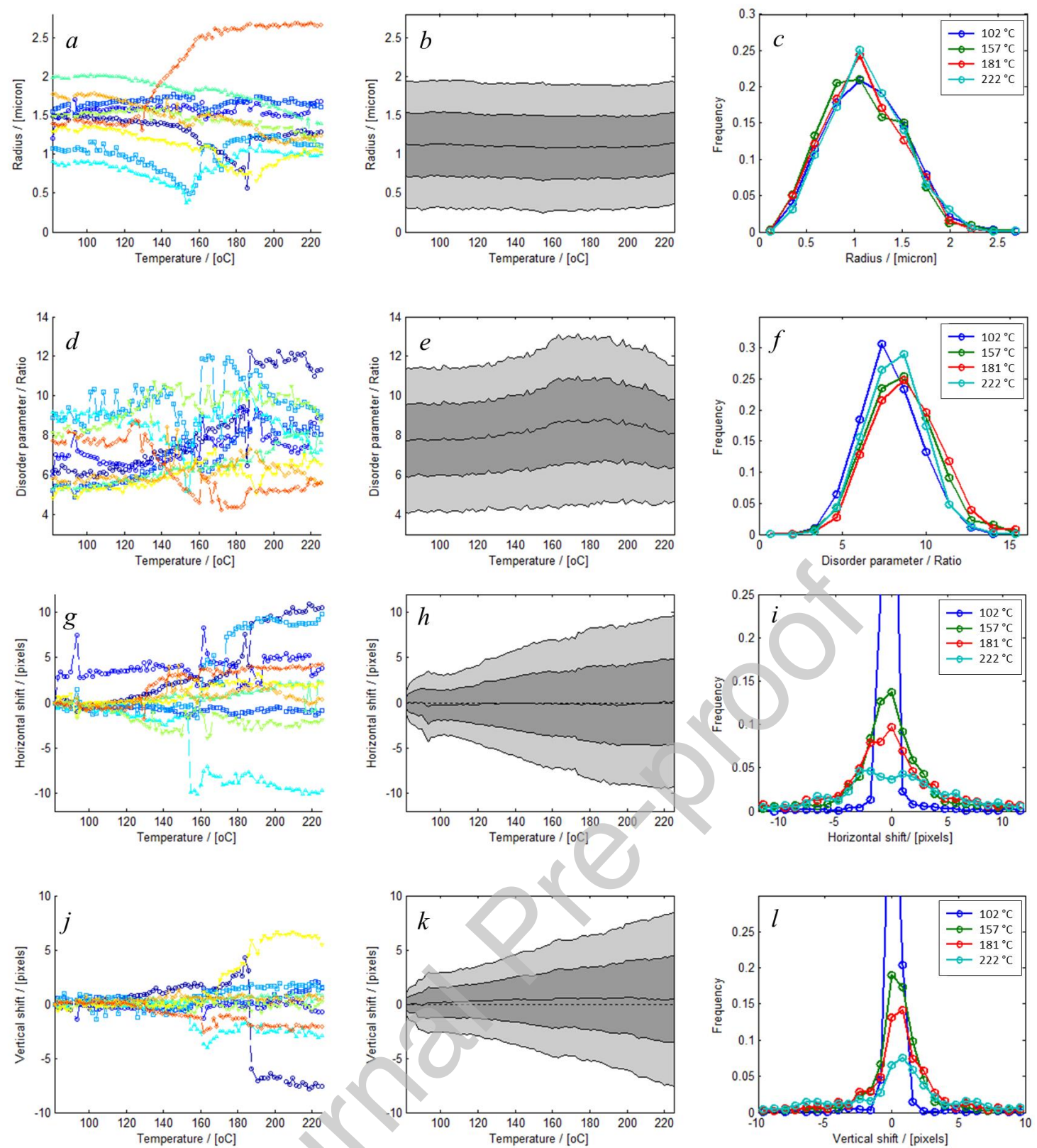

Figure 2. Statistics of parameters characterizing the individual subgrains. From top to bottom rows represent equivalent radius, disorder parameter, and horizontal and vertical shifts of the diffraction spots. Left: evolution of 10 randomly selected subgrains. Middle: Mean and 1 and 2 standard deviations. Right: Distributions for four selected temperatures. The statistics for the first two rows represent all $\sim 2000$ subgrains at each temperature, while the statistics for the latter two rows represent the 498 subgrains that can be tracked through the entire temperature range.

[ Figure 2 in the legend has been added degrees, and indices $a, b, \ldots$ are added to the sub-figures] 

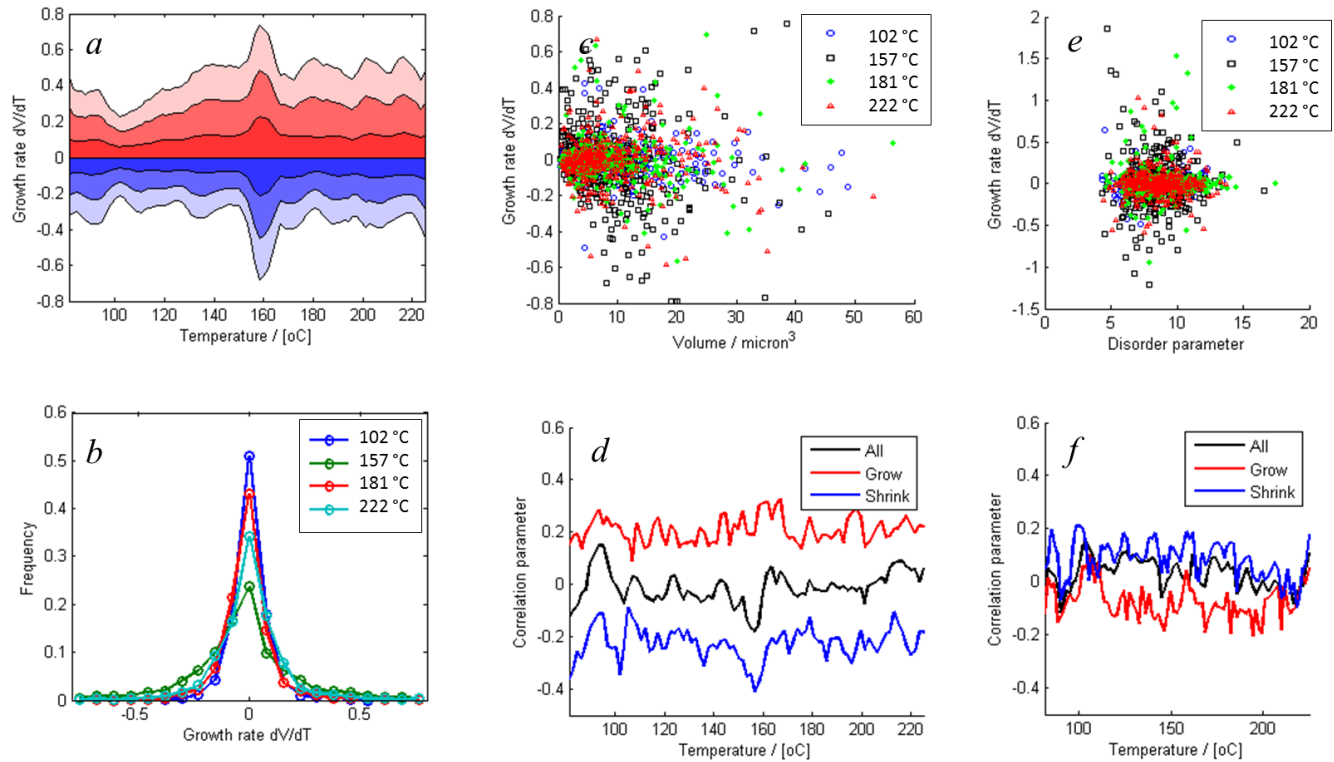

Figure 3. Statistics of growth rates for 498 subgrains. a) Mean growth rates as function of temperature. Red: growing subgrains, blue: shrinking subgrains. The lines closest to zero are mean growth rates, followed by 1 and 2 standard deviations, respectively. b) Distribution of growth rates for four selected temperatures. c) Growth rate versus size at the same four temperatures. d) Correlation between size and growth rate as a function of temperature for all, growing and shrinking subgrains respectively. e) Growth rate versus disorder parameter. f) Correlation between disorder and growth rate as a function of temperature for all, growing and shrinking subgrains respectively.

[ Figure 3 has been added degrees and indices $a, b, \ldots$ as well as two new figures have been added, $3 e$ and $3 f$ ]

\section{References}

[1] M. Cook, T. L. Richards, Observations on the rate and mechanisms of recrystallization in copper, J. Inst. Met. 73 (1946) 1-31.

[2] E. Nes, J. A. Sæter, Recovery, modelling and experiments in N Hansen et al. (Eds), Proceedings $16^{\text {th }}$ Risø Int. Symp. On Mat. Sci.: Microstructure and Crystallographic Aspects of Recrystallization, Risø Denmark, 1995, 169-192.

[3] R. E. Sanders, S. F. Baumann, H. C. Stumpf, Non-heat treatable aluminium alloys in Int. Conf. aluminium alloys (ICAA1), Virginia USA, 1986, 1441.

[4] T. Furu, R. Ørsund, E. Nes, Subgrain growth in heavily deformed aluminium, experimental investigation and modelling treatment, Acta Met. et Mater., 43 (1995), 2209-2232.

[5] R. D. Doherty, D. A. Hughes, F. J. Humphreys, J. J. Jonas, D. Juul Jensen, M. E. Kassner, W. E. King, T.R. McNelley, H. J. McQueen, A. D. Rollett, Current issues in recrystallization: a review, Mat. Sci. Eng. A 238 (1997) 219-274.

[6] T. B. Yu, Deformation microstructure and recovery, Submitted to Recrystallization: Types, Techniques and Applications (Nova Science Publishers).

[7] F. J. Humphreys, A. D. Rollett, G. S. Rohrer, Recrystallization and related annealing phenomena, third ed, Elsevier, Amsterdam, 2017.

[8] T. B. Yu, N. Hansen, X. Huang, Recovery by triple junction motions in aluminium deformed to ultra high strains, Proc. Royal. Soc. A, 467 (2011) 3039-3065.

[9] T. B. Yu, D. A. Hughes, Strong pinning of triple junction migration for robust high stain nanostructures, Phil Mag, (2019) DOI:10.1080/14786435.2018.1562282.

[10] D. Kuhlmann, Zur Theorie der nachwirkungser-scheinungen, Z. f. Phys, 124 (1948) 468-481.

[11] R.A.Vandermeer N.Hansen. Recovery kinetics of nanostructured aluminium: Model and experiment. Acta Mater 56 (2008) $5719-5727$.

[12] T. B. Yu, N. Hansen, Coarsening kinetics of fine scale microstructures in deformed materials, Acta Mater 120 (2016) 40-45.

[13] R. A. Vandermeer, B. B. Rath, Modelling recrystallization kinetics in a deformed iron single crystal, Met. Trans. A, 20A (1989) 391-401.

[14] F. X. Lin, Y. B. Zhang, W. Pantleon, D. Juul Jensen, Importance of non-uniform boundary migration for recrystallization kinetics, Met Mat Trans A, 49 (2018) 5246-5258.

[15] D. Juul Jensen, Growth rates and misorientation relationships between growing nuclei/grains and the surrounding deformed matrix during recrystallization. Acta Met. Mater. 43 (1995) 4117-4129.

[16] H.F. Poulsen, An introduction to three-dimensional X-ray diffraction microscopy. J. Appl. Cryst. 45 (2012) 1084-1097. 
[17] E. M Lauridsen, D. Juul Jensen, H. F. Poulsen, U. Lienert, Kinetics of individual grains during recrystallization, Scripta Mater 43 (2000) 561-566.

[18] E. M. Lauridsen, H. F. Poulsen, S. F. Nielsen, D. Juul Jensen, Recrystallization kinetics of individual bulk grains in $90 \%$ cold-rolled aluminium. Acta Mater 51 (2003) 4423-4435.

[19] S. O. Poulsen, E. M. Lauridsen, A. Lyckegaard, J. Oddershede, C. Gundlach, C. Curfs, D. Juul Jensen, Inside measurements of growth rates and grain-averaged activation energies of individual grains during recrystallization of 50\% cold-rolled aluminium. Scripta Mater. 64 (2011) 1003-1006.

[20] H. O. Sørensen, S. Schmidt, J. P. Wright, G. B. M. Vaughan, S. Techert, E.F. Garman, J. Oddershede, J. Davaasambuu, K. S. Paithankar, C. Gundlach, H. F. Poulsen, Multigrain crystallography. Z. Kristallogr. 227 (2012) 63-78.

[21] C. Gundlach, W. Pantleon, E.M. Lauridsen, L. Margulies, R. D. Doherty, H. F. Poulsen, Direct observation of subgrain evolution during recovery of cold-rolled aluminium. Scripta mater. 50 (2004) 477-481.

[22] T. Martin and A. Koch. Recent development in X-ray imaging with micrometer spatial resolution, J. Synchr. Rad. 13 (2006) 180-194.

[23] A. C.Jakobsen, H. Simons, W. Ludwig, C. Yildirim, H. Leemreize, L. Porz, C. Detlefs, H. F. Poulsen, Mapping of individual dislocations with dark field x-ray microscopy. J. Appl. Cryst. 52 (2019) 122-132.

[24] H. Simons, A. King, W. Ludwig, C. Detlefs, W. Pantleon, S. Schmidt, F. Stöhr, I. Snigireva, A. Snigirev, H. F. Poulsen, Dark-field X-ray microscopy for multiscale structural characterization. Nature Comm, 6 (2015) 6098.

[25] H. Simons, A.B. Haugen, A. C.Jakobsen, S; Schmidt, F. Stöhr, M. Majkut, C. Detlefs, J. E: Daniels, D. Damjanovic, H. F. Poulsen, Longrange symmetry breaking in embedded ferroelectrics. Nature Mater. 17 (2018) 814-819.

[26] B.C. Larson, W. Yang, G.E. Ice, J.D. Budai, J.Z.Tischler, Three-dimensional X-ray structural microscopy with submicrometre resolution. Nature 415 (2002) 887-90.

[27] Y.B.Zhang, J.D. Budai, J.Z.Tischler, W. Liu, R. Xu, E.R. Homer, A. W. Godfrey, D. Juul Jensen, Boundary migration in a 3D deformed microstructure inside an opaque sample. Scientific Reports 7 (2017) 4423.

[28] C. Xu, Y.B. Zhang, A. W. Godfrey, G. Wu, W. Liu, J.Z. Tischler, Q. Liu, D. Juul Jensen, Direct observation of nucleation in the bulk of an opaque sample. Scientific Reports 7 (2017) 42508.

[29] Q. Xing, X. Huang, N. Hansen, Recovery of heavily cold rolled aluminium: Effect of local texture. Metall. Mat. Transactions A, 37A (2006) $1311-1322$

[30] A. Albou, A. Borbely, C. Maurice, J. H. Driver, Orientation-dependent recovery in strongly deformed Al-0.1\% Mn crystals. Phil. Mag.,91 (2011) 3981-4000.

[31] M. Ferry, F.J. Humphreys, Onset of abnormal subgrain growth in cold rolled $\left\{\begin{array}{ll}1 & 10\end{array}<001>\right.$ oriented copper single crystals. Mater. Sci. Engineer. A 435 (2006) 447-452.

[32] T. Hasegawa, T. Yakou, U.F. Kocks, Length changes and stress effects during recovery of deformed aluminium. Acta Mettall. 30 (1982), 235-243.

[33] S.R. Ahl, H. Simons, Y. Zhang, C. Detlefs, F. Stöhr, A.C. Jakobsen, D. Juul Jensen, H.F. Poulsen, Ultra-low-angle boundary networks within recrystallizing grains. Scripta Materialia 139 (2017) 87-91.

[34] Y.B. Zhang, Quantification of local boundary migration in 2D/3D. $40^{\text {th }}$ Risø International Symposium on Materials Science, IOP Conf. Series: Materials Science and Engineering 580 (2019) 012015, doi:10.1088/1757-899X/580/1/012015. 


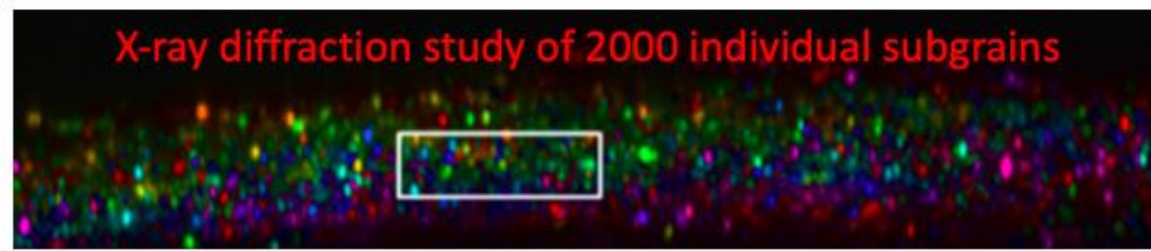

Growth of 10 subgrains No relation between growth rate and size
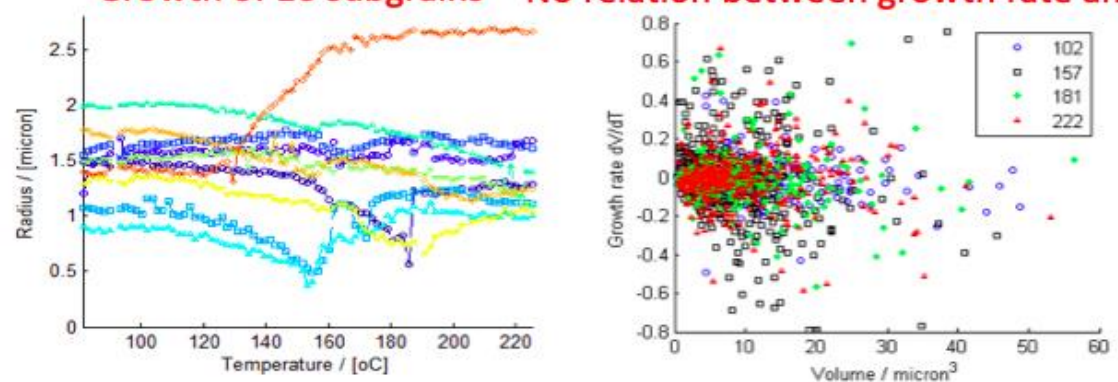